\title{
Discharge from the Emergency Department and Early Hospital Revaluation in Patients with COVID- 19 Pneumonia: an Observational Study
}

Massimo Mattioli ( $\sim$ massimo.mattioli@ospedalimarchenord.it)

Azienda Ospedaliera Ospedali Riuniti Marche Nord https://orcid.org/0000-0002-2118-7260

Devis Benfaremo

Azienda Ospedaliero Universitaria Ospedali Riuniti di Ancona Umberto I G M Lancisi G Salesi, Università Politecnica delle Marche: Universita Politecnica delle Marche

\section{Francesca Fulgenzi}

Azienda Ospedaliera Ospedali Riuniti Marche Nord, Azienda Ospedaliera Ospedali Riuniti Marche Nord

\section{Silvia Gennarini}

Azienda Ospedaliera Ospedali Riuniti Marche Nord, Azienda Ospedaliera Ospedali Riuniti Marche Nord

Luciano Mucci

Azienda Ospedaliera Ospedali Riuniti Marche Nord, Azienda Ospedaliera Ospedali Riuniti Marche Nord

Margherita Lambertini

Azienda Ospedaliera Ospedali Riuniti Marche Nord, Azienda Ospedaliera Ospedali Riuniti Marche Nord

Francesca Padiglione

Azienda Ospedaliera Ospedali Riuniti Marche Nord, Azienda Ospedaliera Ospedali Riuniti Marche Nord

Ramona Brugnera

Azienda Ospedaliera Ospedali Riuniti Marche Nord, Azienda Ospedaliera Ospedali Riuniti Marche Nord

Barbara Gabrielli

Azienda Ospedaliera Ospedali Riuniti Marche Nord, Azienda Ospedaliera Ospedali Riuniti Marche Nord Flavia Giorgino

Azienda Ospedaliera Ospedali Riuniti Marche Nord, Azienda Ospedaliera Ospedali Riuniti Marche Nord Maria Capalbo

Azienda Ospedaliera Ospedali Riuniti Marche Nord, Azienda Ospedaliera Ospedali Riuniti Marche Nord

\section{Edoardo Berselli}

Azienda Ospedaliera Ospedali Riuniti Marche Nord, Azienda Ospedaliera Ospedali Riuniti Marche Nord

\section{Gianluca Moroncini}

Azienda Ospedaliero Universitaria Ospedali Riuniti di Ancona Umberto I G M Lancisi G Salesi, Universita Politecnica delle Marche

\section{Gabriele Frausini}

Azienda Ospedaliera Ospedali Riuniti Marche Nord, Azienda Ospedaliera Ospedali Riuniti Marche Nord Umberto Gnudi 


\section{Original research}

Keywords: COVID-19, pneumonia, outpatient, emergency department

Posted Date: March 11th, 2021

DOI: https://doi.org/10.21203/rs.3.rs-289317/v1

License: (c) (i) This work is licensed under a Creative Commons Attribution 4.0 International License. Read Full License 


\section{Abstract}

Background Health National Systems world-wide are facing the severe acute respiratory syndrome coronavirus 2 (SARS-CoV-2) pandemic. We purpose an outpatient management for patients affected by SARS-CoV-2 related pneumonia at risk of progression, after discharge from Emergency Department (ED).

Methods This was a single-center prospective observational study. We enrolled patients with confirmed SARS-CoV-2 pneumonia, without hypoxemic respiratory failure, and at least one of the following: age $\geq$ 65 or presence of one or more comorbidities or pneumonia involvement $>25 \%$ on high resolution computed tomography (HRCT). The ambulatorial visit was performed after at least 48 hours, then patients could be discharged, admitted for hospitalization, or deferred for a further visit. As a control, we evaluated a historical cohort of patients hospitalized with comparable clinical and radiological features.

Results A total of 84 patients were enrolled ( $51 \mathrm{M}$, mean age $62.8 \mathrm{y})$. Two-thirds of patients had at least one comorbidity and $41.6 \%$ had a lung involvement $>25 \%$ at HRCT; the mean duration of symptoms was $8 \pm 3$ days and the mean $\mathrm{PaO} 2 / \mathrm{FiO} 2$ ratio $357.5 \pm 38.6$. At the end of the follow-up period, 69 patients had been discharged and 15 hospitalized (mean stay 6 days). Older age and higher NEWS2 were significant predictors of hospitalization at the first follow-up visit. One hospitalized patient died of septic shock. In the control group, the mean hospital stay was 8 days.

Conclusions Adopting a "discharge and early revaluation" strategy appear to be safe and feasible. This approach may help to optimize hospital resources during the SARS-CoV-2 pandemic.

\section{Background}

Severe Acute Respiratory Syndrome Coronavirus 2 (SARS-CoV-2) has been first identified in December 2019 in China [1], where it caused the first cluster of cases that have rapidly diffused worldwide, leading to a pandemic. Italy is one of the most affected countries with more than 2.7 million cases and a case fatality rate of $3.4 \%[2]$.

The Italian National Health System, like most countries, has struggled to withstand the epidemic wave, especially due to the lack of hospital beds, both for ordinary and intensive care.

In a large epidemiological study, only $19 \%$ of patients with SARS-CoV-2 infection developed severe or critical illness and pneumonia with hypoxemic respiratory failure is the more frequent complication [3]. Several risk scores adopting clinical, radiological and laboratory markers of progression of the disease [4, 5] that could help in the management of patient affected by Coronavirus 19 disease (COVID-19) have been proposed; however, their real-life applicability is limited, as they must be integrated into a more complex clinical judgment.

Indeed, one of the crucial decisions is the discharge from the Emergency Department (ED). In a retrospective cohort of low-risk patients (median age 38 and 61\% without comorbidities) with COVID19- 
like symptoms discharged from ED, the readmission rate was $4 \%$ after a median of 5 days [6]. In a multicentre study enrolling only confirmed COVID-19 patients, about $5 \%$ of patients discharged from ED was readmitted, and then hospitalized, within 72 hours and $8.2 \%$ within seven days; the major predictors of readmission were the presence of hypoxia, fever and age $>60$ years [7].

A useful solution for the optimization of resources may be to treat and follow-up these patients as outpatient. Two Spanish recent studies reported similar experiences [8, 9]. Teigell Muñoz et al

We consider that a goal should be to identify at their first access to ED symptomatic patients with mild-tomoderate COVID-19 pneumonia and reserving them a fast-track access to hospital care, if needed. In this prospective study aims to evaluate the feasibility and safety of our new protocol in patients with SARSCoV-2 related pneumonia discharged from the ED at risk of progression.

\section{Materials And Methods}

\section{Study design, setting and selection of population}

The study population was a cohort of patients admitted and discharged within a few hours from the Emergency Department of Azienda Ospedaliera Ospedali Riuniti Marche Nord (Pesaro), Italy and reevaluated in our COVID-19 Early Medical Revaluation Ambulatory. All the patients were older than 18 and gave informed consent. The inclusion criteria were: 1) confirmed SARS-CoV-2 infection by RT-PCR nasopharyngeal swab; 2) age $\geq 65$ or presence of at least one comorbidity including hypertension, chronic obstructive pulmonary disease (COPD), chronic heart failure, obesity (BMI > 30), active neoplasia or semiquantitative pneumonia involvement $>25 \%$ on HRCT; 3 ) radiological evidence of interstitial pneumonia at HRCT with semiquantitative extension $\leq 50 \%$ [8]; 4) absence of functional respiratory failure defined by the presence of both $\mathrm{PaO} 2 / \mathrm{FiO}_{2} \geq 300$ and no desaturation at quick walking test (QWT) [9]. The patients who did not meet all the inclusion criteria were excluded.

As a control group, we collected clinical data of a historical cohort of patients admitted to the same hospital from March 10 to December 31, 2020 for COVID-19 pneumonia. Controls were matched at admission to ED for age, sex, number of comorbidities, evidence of pneumonia at HRCT and absence of respiratory failure (considered as $\mathrm{PaO} 2 / \mathrm{FiO} 2$ ratio $>300$ ).

The study was performed according to the ethical guidelines of the Declaration of Helsinki (seventh revision). The report is according to the Strengthening the Reporting of Observational Studies in Epidemiology (STROBE) Statement for observational studies.

\section{Interventions and measurements}

Upon discharge, clinicians gave a pulse oximeter and a personal diary to measure and report three times daily i) body temperature, ii) peripheral oxygen saturation (SpO2) at rest and iii) after a QWT. The ambulatorial visit was performed after at least 48 hours after discharge and included: complete physical examination, personal diary check, lung ultrasound [10], arterial blood gas test and quick walking test. 
After the visit, patients could be discharged, admitted for hospitalization, or deferred for a further visit. The last follow-up of all discharged patients was a phone call made 30 days after the index admission.

\section{Data analysis}

Continuous variables were summarized using mean \pm standard deviation (SD) or median and interquartile range (IQR) if the data were parametric or nonparametric, respectively. Categorical data were summarized as absolute numbers and percentages. The results were tested for normality by KolmogorovSmirnov's test. Student's t-test or nonparametric Mann-Whitney $U$ test were used to compare continuous variables in subgroups of patients, as appropriate. Chi square test was used to compare categorical variables. A logistic regression model was performed to identify variables associated with hospitalization. A p value $<0.05$ was considered significant. All statistical analyses were performed with the STATA Software.

\section{Results}

\section{Characteristics of study subjects}

From November 15th, 2020 to January 10th, 2021, 84 patients were enrolled (51 M, mean age $62.8 \pm 12.0$ years). Table 1 summarizes the baseline characteristics of the cohort. Most patients had one or more comorbidities (22.6\% were obese, $23.8 \%$ had diabetes, $32.1 \%$ hypertension, $8.3 \%$ COPD). 
Table 1

Baseline characteristics of the enrolled patients

\begin{tabular}{|c|c|}
\hline Age & $62.8 \pm 12.0$ \\
\hline Gender (M:F) & $51: 33$ \\
\hline \multirow[t]{7}{*}{ Number and type of comorbidities } & Mean number $1 \pm 0.8$ \\
\hline & Hypertension 27 (32.1\%) \\
\hline & Obesity 19 (22.6\%) \\
\hline & Diabetes $20(23.8 \%)$ \\
\hline & COPD 7 (8.3\%) \\
\hline & Neoplasms 3 (3.6\%) \\
\hline & Immunodepression 2 (2.4\%) \\
\hline Days from symptoms onset & $8.0 \pm 3.0$ \\
\hline \multirow[t]{6}{*}{ Reported symptoms } & Fever $81(96.4 \%)$ \\
\hline & Cough $49(58.3 \%)$ \\
\hline & Shortness of breath 29 (34.5\%) \\
\hline & Fatigue 20 (23.8\%) \\
\hline & GI symptoms 17 (20.2\%) \\
\hline & Anosmia/ageusia 11 (13.1\%) \\
\hline Pulse oximetry (\%) & $96.8 \pm 1.5$ \\
\hline $\mathrm{PaO} 2 / \mathrm{FiO} 2$ ratio & $357 \pm 38$ \\
\hline Respiratory rate (breaths/min) & $16.3 \pm 1.8$ \\
\hline $\mathrm{HRCT}>25 \%$ & $35(41.7 \%)$ \\
\hline C-reactive protein $(\mathrm{mg} / \mathrm{l})$ & $49.1 \pm 25.2$ \\
\hline NLR & $5.5 \pm 4.6$ \\
\hline D-dimer (mg/l) & $877 \pm 805$ \\
\hline
\end{tabular}

At admission, the mean duration of symptoms attributable to SARS-CoV-2 infection was $8.0 \pm 3.0$ days. The most common reported symptoms were fever (96.4\%), cough (58.3\%), shortness of breath (34.5\%), fatigue (23.8\%), anosmia/ageusia (13.1\%) and gastrointestinal involvement $(20.2 \%)$. The mean 
peripheral oxygen saturation was $96.8 \pm 1.5 \%$, the mean respiratory rate $16.3 \pm 1.8$ per minute, and the mean $\mathrm{PaO} 2 / \mathrm{FiO} 2$ ratio $357.5 \pm 38.6$. A significant proportion of patients had an involvement $>25 \%$ at HRCT (41.6\%). All patients were discharged after a mean ED stay of $16.3 \pm 14.6$ hours, with the abovementioned instructions and a scheduled follow-up visit.

\section{Main results}

Six (6) patients (7.1\%) were re-hospitalized before the planned visit ( $5 \mathrm{M}$, mean age $67.6 \pm 9.04 \mathrm{y})$ after a mean $1.3 \pm 0.5$ days at home. Of the 78 patients that attended the first follow-up visit after a mean $3.3 \pm$ 1.1 days from the index admission, only 1 had to start oxygen support at home. The peripheral oxygen saturation was $96.6 \pm 1.8 \%$, the respiratory rate $16.5 \pm 3.7$ per minute, and the mean $\mathrm{PaO} 2 / \mathrm{FiO} 2$ ratio $375.3 \pm 55.1$. Most patients were receiving heparin $(82 \%)$ and steroids $(74.3 \%)$. The majority of patients filled out the diary (88.4\%), but only in $13 / 69$ diaries warning signs were noted (18.8\%). Four patients $(5.13 \%)$ had to be admitted, 23 (29.5\%) were deferred for a further visit and 51 (65.4\%) were definitely discharged from hospital care. Of the 23 patients that had been referred to a second visit, $4(17.4 \%)$ had to be admitted before the planned follow-up after a mean $4.5 \pm 1.9$ days. Of the 19 patients that attended the second visit after a mean of $7.4 \pm 2.3$ days from the index admission, only $2(10.5 \%)$ needed oxygen supplementation at home. The majority of patients were still on heparin (73.7\%) and steroids (68.4\%). At the end of the hospital evaluation, 14 (73.6\%) patients were definitely discharged, 1 admitted for persistent fever and 4 further deferred for another follow-up visit. Of the 17 patients that were managed in more than one follow-up visit, 14 showed a reduction trend or, at least, stationary in the lung ultrasonographic score. All patients were finally discharged after the third hospital visit. The mean duration of the ambulatorial management was $4.6 \pm 2.7$ days. Details of management of the single patients are summarized in Figs. 1 and 2.

At the 30-day follow-up call, all but one patients were alive. The deceased patient was a 84-year-old woman that had to be admitted after the second visit because of hypoxemic respiratory failure. After a hospitalization that lasted 12 days ( 6 in the Intensive Care Unit and 6 in the Sub-intensive Care Unit), she was discharged home. Two days later she was referred to ED for fever, chills, myalgias and shortness of breath. The investigations showed the presence of Enterococcus Faecium in the urine and Acinetobacter Baumannii in the lower respiratory tract. She died of septic shock after 36 hours.

A total of $15 / 84(17.8 \%)$ patients had to be admitted by the end of the follow-up window. The mean duration of hospitalization was $6.1 \pm 2.5$ days. All but 3 institutionalized patients (one still receiving low flow oxygen supplementation) were at home at the time of the phone call. The 66 patients that could answer were also asked to rate the ambulatory service on a 10-point scale, with 10 representing the best possible. Overall, they rated the service with a mean of $9.1 \pm 1.5$ points.

Table 2 summarizes the comparisons between patients that have been hospitalized and patients that were managed home, considering the variables at the index admission and at the first follow-up visit. Overall, there were no differences between the two groups at the index admission except for age (higher for the hospitalized group). Conversely, considering only patients that attended the first follow-up visit ( $n$ 
= 78), the group that had been hospitalized showed higher mean NEWS2, lower peripheral oxygen saturation at rest and after a walking test, lower $\mathrm{PaO} 2 / \mathrm{FiO} 2$ ratio and, more importantly, their diary was more likely to be positive for warning signs while at home. 
Table 2

Difference between patients that have been hospitalized or patients managed at home, at the index admission ( $n=84$ ) or the first follow-up admission ( $n=78$ )

\begin{tabular}{|c|c|c|c|}
\hline INDEX ADMISSION & $\begin{array}{l}\text { Hospitalized patients }(n= \\
\text { 15) }\end{array}$ & $\begin{array}{l}\text { Home-managed patients }(n= \\
69)\end{array}$ & $p$ \\
\hline Age & $69.5 \pm 10.6$ & $61.5 \pm 11.9$ & .01 \\
\hline Male & $10(66.7 \%)$ & $41(59.4 \%)$ & ns \\
\hline Number of comorbidities & $1.2 \pm 0.7$ & $0.9 \pm 0.8$ & ns \\
\hline Days from symptoms onset & $8.4 \pm 2.7$ & $8 \pm 2.1$ & ns \\
\hline NEWS2 & $0.8 \pm 0.7$ & $0.7 \pm 0.8$ & ns \\
\hline Pulse oximetry (\%) & $97.2 \pm 1.5$ & $96.8 \pm 1.5$ & ns \\
\hline $\begin{array}{l}\text { Post-effort pulse oximetry } \\
(\%)^{\star}\end{array}$ & $95.7 \pm 1.5$ & $95.3 \pm 1.9$ & ns \\
\hline $\mathrm{PaO} 2 / \mathrm{FiO} 2$ ratio & $356.5 \pm 33.5$ & $357.8 \pm 39.9$ & ns \\
\hline HRCT > 25\% & $5(33.3 \%)$ & $30(43.5 \%)$ & ns \\
\hline C-reactive protein (mg/l) & $4.0 \pm 3.4$ & $5.1 \pm 3.6$ & ns \\
\hline NLR & $6 \pm 5.7$ & $5.4 \pm 4.4$ & ns \\
\hline D-dimer (mg/l) & $728 \pm 485$ & $910 \pm 859$ & ns \\
\hline FOLLOW-UP ADMISSION & $\begin{array}{l}\text { Hospitalized patients }(n= \\
\text { 9) }\end{array}$ & $\begin{array}{l}\text { Home-managed patients }(\mathrm{n}= \\
69)\end{array}$ & $\mathbf{p}$ \\
\hline Age & $70.6 \pm 11.9$ & $61.5 \pm 11.9$ & .03 \\
\hline Male & $5(55.6 \%)$ & $41(59.4 \%)$ & ns \\
\hline Number of comorbidities & $1.1 \pm 0.7$ & $0.9 \pm 0.8$ & ns \\
\hline Days from symptoms onset & $11.4 \pm 3$ & $11.3 \pm 3.4$ & ns \\
\hline NEWS2 & $1.5 \pm 1.6$ & $0.2 \pm 0.5$ & $<.001$ \\
\hline Pulse oximetry (\%) & $94 \pm 2$ & $97 \pm 1.5$ & $<.001$ \\
\hline $\begin{array}{l}\text { Post-effort pulse oximetry } \\
(\%)^{*}\end{array}$ & $91.4 \pm 3.4$ & $95.7 \pm 2.4$ & $<.001$ \\
\hline $\mathrm{PaO} 2 / \mathrm{FiO} 2$ ratio & $314 \pm 36$ & $384 \pm 51$ & $<.001$ \\
\hline HRCT > 25\% & $2(22.2 \%)$ & 30 (43.5\%) & ns \\
\hline
\end{tabular}

NEWS2 Nationally Early Warning Score 2; HRCT high-resolution computed tomography of the chest. Data were analysed by $t$ test or chi square as appropriate. *measured after Quick Walking Test; §Only 69 patients filled out the diary as instructed. 


\section{INDEX ADMISSION}

Warning at the diary§
Hospitalized patients $(\mathrm{n}=$

15)

$6(85.7 \%)$
Home-managed patients $(\mathrm{n}=$

69) p

$7(11.3 \%)$

$<.001$

NEWS2 Nationally Early Warning Score 2; HRCT high-resolution computed tomography of the chest. Data were analysed by $t$ test or chi square as appropriate. *measured after Quick Walking Test; §Only 69 patients filled out the diary as instructed.

At univariate analysis, no variables were significant predictors of hospitalization within the follow-up period at the index ED admission, except for age (OR 1.06, 95\% $\mathrm{Cl} 1.01-1.11)$. Considering the first follow-up visit, age (OR 1.11, 95\% $\mathrm{Cl} 1.00-1.23$ ) and NEWS2 (OR 7.76, 95\% $\mathrm{Cl} 1.76-36.1$ ) were significant predictors of hospitalization after adjusting for gender and number of comorbidities (Table 3 ).

Table 3

Results of the logistic regression model: predictors of hospital admission at the first follow-up visit $(n=78)$

\begin{tabular}{|llllll|}
\hline Variable & OR & $\mathbf{9 5 \%} \mathbf{C l}$ & St. err & $\mathbf{Z}$ & $\mathbf{P}$ \\
\hline Age & $\mathbf{1 . 1 1}$ & $1.00-1.23$ & 0.05 & 2.09 & 0.037 \\
\hline Gender (M) & 0.50 & $0.04-5.58$ & 0.62 & -0.55 & 0.579 \\
\hline Number of comorbidities & 0.51 & $0.15-1.72$ & 0.31 & -1.07 & 0.283 \\
\hline NEWS2 & $\mathbf{7 . 7 6}$ & $1.66-36.1$ & 6.09 & 2.61 & 0.009 \\
\hline NEWS2 Nationally Early Warning Score 2; OR Odds Ratio \\
\hline
\end{tabular}

We next compared the group of patients that have been managed with the Early Discharge and ReEvaluation protocol and a historical cohort of patients that were admitted to the ED and hospitalized, marched for age, sex, number of comorbidities, evidence of pneumonia at HRCT and absence of respiratory failure (considered as $\mathrm{PaO} 2 / \mathrm{FiO} 2$ ratio $>300$ ). The cohorts were comparable for all other variables (data not shown). Although the mean duration of hospitalization was not different between groups (6.2 \pm 5.5 vs $8.8 \pm 5.2$ days), in our cohort only $15 / 84$ (17.8\%) patients were hospitalized, compared to the totality of the 63 patients of the historical cohort.

\section{Discussion}

Discharging patients with SARS-CoV-2 pneumonia and without hypoxia and managing them in an ambulatorial service appears to be safe and feasible.

Of the 84 enrolled patients, all completed the follow-up period. Only one died of septic shock due to a nosocomial infection; hospitalization was needed for the progressive development of hypoxia detected in 
a control visit. This cohort of patients was representative of those with SARS-CoV-2 related pneumonia with low-to-moderate risk for progression of the disease [4, 11]: $60.7 \%$ of them were male, the median age was 62.8 years, with 1 or more comorbidities and $41.7 \%$ of them had a lung involvement higher the $25 \%$ (but lower than $50 \%$ ) at HRCT score. Fever was reported by almost all patients $(96.4 \%)$, cough by more than a half $(58.3 \%)$ and shortness of breath by a third of them (34.5\%).

COVID-19 has a triphasic course: the early disease (I), the pulmonary (Ila without and Ilb with hypoxia) and the hyperinflammation phases [12]. With the ambulatorial strategy, we tried to revaluate patients between stage Ila and Ilb. In fact, our patients complained of symptoms attributable to SARS-CoV-2 infection from $8.0 \pm 3.0$ days and the mean duration of the ambulatorial management was $4.6 \pm 2.7$ days.

At the index admission, the respiratory status was satisfactory: all patients were eupnoeic, with a negative QWT and values of $\mathrm{PaO} 2 / \mathrm{FiO} 2$ ratio at the lower boundaries for the need of oxygen supplementation. Older age and higher NEWS2 appear to be the most important predictors of hospitalization. However, of the 10 patients that had been admitted by the end of the first scheduled follow-up, only 2 had a NEWS2 score $\geq 2$ at the index admission, whereas all the 4 patients that presented to the first visit with a NEWS2 score $\geq 2$ were hospitalized. A small prospective Norwegian study claimed that the NEWS2 score predicted severe disease and in-hospital mortality better than the other risk score evaluated [13], while in a larger multicentric cohort, the model of 'NEWS2 + age' alone had poor-to-moderate discrimination for severe COVID-19 at 14 days (AUC 0.7), that improves with the integration of other blood and physical parameters [14]. With these limitations, this is the first study to assess the utility of a longitudinal evaluation of NEWS2 score for outpatients with COVID-19.

Importantly, a high proportion of patients filled out the diary: it suggests satisfactory training during the enrolment, good performance status and/or a correct engagement of the caregiver. This tool proved to be useful and helped physicians during the follow-up visit to decide how to manage the patient: in our cohort, $85.7 \%$ of patients hospitalized after a control visit had warning signs (persistent fever $>38.5^{\circ} \mathrm{C}$ or pulse oximetry lower than $92 \%$ at rest or lower than $90 \%$ after a QWT), versus the $11.3 \%$ that were not hospitalized. For COVID-19 patients, the presence of a caregiver or social support is crucial due to the risk of progression; in our cohort, the lack of home assistance represented the reason for hospitalization in 2 out of 15 patients.

Unsurprisingly, there was no difference between the patients managed home and those hospitalized with regard to lung involvement at HRCT. Although radiological abnormalities have prognostic relevance in patients with extensive involvement $[5,15]$, in the Diamond Princess Ship study half of asymptomatic patients had pathologic findings at HRCT [16], suggesting a limited specificity of this technique. Thus, HRCT extension alone may not be a good predictor of worse outcomes in patients presenting at the ED without respiratory failure. In this context, we used lung ultrasound, that is a safe and reproducible tool for the longitudinal evaluation of the severity of pneumonia [17], as described.

During a pandemic, the optimization of resources is crucial. Although we were able to provide only an indirect comparison with a cohort of comparable patients, adopting a "discharge and early revaluation" 
strategy may have spared many hundreds of hospitalization-days, considering that without the ambulatorial management most, if not all, of the enrolled patients would have been hospitalized. This is also more important because, to date, no therapies have proved to be effective for SARS-CoV-2-related pneumonia patients without respiratory failure [18], even if hospitalized.

\section{Limitations}

The study was observational and conducted in a single center with a small cohort of patients, so data should not be generalizable especially regarding organizational aspects and territorial management (our hospital was the hub center for COVID-19). The inclusion criteria were established with a multiparametric evaluation that may have determined selection and information bias. There were no absolute indications for the hospitalization in the control visit but it was left to the medical judgment. The control group was searched in a historical cohort to match low-risk patients that were managed as in-patient, but this determined a selection of patients and an incomplete matching of data.

\section{Conclusions}

Our study demonstrates that COVID-19 patients without respiratory failure can be safely managed with a "discharge and early revaluation" approach directly from the ED staff. This translates into similar outcomes for the patients but with a significant saving of resources for the National Health System. Further studies are needed to assess the best management strategy for low-to-moderate risk COVID-19 patients.

\section{List Of Abbreviations}

SARS-CoV-2 Severe acute respiratory syndrome coronavirus 2

ED Emergency Department

COVID-19 Coronavirus 19 disease

HRCT High-resolution computed tomography

COPD Chronic obstructive pulmonary disease

QWT Quick walking test

\section{Declarations}

Ethics approval and consent to participate: The local ethics committee waived approval of the study given our outpatient service was ongoing for the pandemic; all the patients gave written informed consent Consent for publication: It was obtained and collected from all the patients 
Availability of data and materials: The datasets used and/or analysed during the current study are available from the corresponding author on reasonable request

Competing interests: All the authors report no conflict of interest

Funding: Azienda Ospedaliera Ospedali Riuniti Marche Nord (Pesaro, Italy) guaranteed the funds for the activation and the implementation of the service

Author contributions: MM, SG, LM, RB, MC and UG conceived the study, designed the trial, and obtained research funding. MM, FF, LM, SG, BG, LM, FP, FG supervised the conduct of the trial, the recruitment of patients and data collection. MM and FF managed the data, including quality control. BD provided statistical advice on study design and analysed the data. MM and BD drafted the manuscript, and all authors contributed substantially to its revision and approved the final version.

Acknowledgements: None

\section{References}

1. Zhu N, Zhang D, Wang W et al. China Novel Coronavirus Investigating and Research Team. A Novel Coronavirus from Patients with Pneumonia in China, 2019. N Engl J Med. 2020 Feb 20;382(8):727733. doi: 10.1056/NEJMoa2001017. Epub 2020 Jan 24. PMID: 31978945; PMCID: PMC7092803.

2. ISS COVID-19 integrated surveillance: Available at: https://www.epicentro.iss.it/en/coronavirus/sarscov-2-dashboard (last accessed February 20, 2021)

3. Wu Z, McGoogan JM. Characteristics of and Important Lessons From the Coronavirus Disease 2019 (COVID-19) Outbreak in China: Summary of a Report of 72314 Cases From the Chinese Center for Disease Control and Prevention. JAMA. 2020 Apr 7;323(13):1239-1242. doi:

10.1001/jama.2020.2648. PMID: 32091533.

4. Liang W, Liang H, Ou L et al. China Medical Treatment Expert Group for COVID-19. Development and Validation of a Clinical Risk Score to Predict the Occurrence of Critical Illness in Hospitalized Patients With COVID-19. JAMA Intern Med. 2020 Aug 1;180(8):1081-1089. doi:

10.1001/jamainternmed.2020.2033. PMID: 32396163; PMCID: PMC7218676.

5. Feng Z, Yu Q, Yao S et al. Early prediction of disease progression in COVID-19 pneumonia patients with chest CT and clinical characteristics. Nat Commun. 2020 Oct 2;11(1):4968. doi:

10.1038/s41467-020-18786-x. PMID: 33009413; PMCID: PMC7532528.

6. Berdahl CT, Glennon NC, Henreid AJ et al. The safety of home discharge for low-risk emergency department patients presenting with coronavirus-like symptoms during the COVID-19 pandemic: A retrospective cohort study. J Am Coll Emerg Physicians Open. 2020 Aug 17;1(6):1380-5. doi: 10.1002/emp2.12230. Epub ahead of print. PMID: 32838391; PMCID: PMC7436406.

7. Margus C, Sondheim SE, Peck NM et al. Discharge in Pandemic: Suspected Covid-19 patients returning to the Emergency Department within 72 hours for admission. Am J Emerg Med. 2020 Aug 
18:S0735-6757(20)30721-X. doi: 10.1016/j.ajem.2020.08.034. Epub ahead of print. PMID: 33046303; PMCID: PMC7434326.

8. Teigell Muñoz FJ, García-Guijarro E, García-Domingo P, Pérez-Nieto G, Roque Rojas F, García-Peña M, Nieto Gallo MA, Melero Bermejo JA, de Guzman García-Monge MT, Granizo JJ. A safe protocol to identify low-risk patients with COVID-19 pneumonia for outpatient management. Intern Emerg Med. 2021 Feb 23:1-9. doi: 10.1007/s11739-021-02660-9. Epub ahead of print. PMID: 33620681; PMCID: PMC7900647.

9. Llorens P, Moreno-Pérez O, Espinosa B, García T, Payá AB, Sola S, Molina F, Román F, Jiménez I, Guzman S, Gil-Rodrigo A, Peña-Pardo B, Merino E, Gil J, San-Inocencio D, Andrés M, Sánchez-Payá J. An integrated emergency department/hospital at home model in mild COVID-19 pneumonia: feasibility and outcomes after discharge from the emergency department. Intern Emerg Med. 2021 Feb 24:1-10. doi: 10.1007/s11739-021-02661-8. Epub ahead of print. PMID: 33625661; PMCID: PMC7903217.

10. Revel MP, Parkar AP, Prosch $\mathrm{H}$ et al. European Society of Radiology (ESR) and the European Society of Thoracic Imaging (ESTI). COVID-19 patients and the radiology department - advice from the European Society of Radiology (ESR) and the European Society of Thoracic Imaging (ESTI). Eur Radiol. 2020 Sep;30(9):4903-4909. doi: 10.1007/s00330-020-06865-y. Epub 2020 Apr 20. PMID: 32314058; PMCID: PMC7170031.

11. Paglia S, Nattino G, Occhipinti F et al. The Quick Walk Test: A Noninvasive Test to Assess the Risk of Mechanical Ventilation During COVID-19 Outbreaks. Acad Emerg Med. 2020 Nov

29:10.1111/acem.14180. doi: 10.1111/acem.14180. Epub ahead of print. PMID: 33249678; PMCID: PMC7753836.

12. Pivetta E, Goffi A, Tizzani M et al. Lung Ultrasonography for the Diagnosis of SARS-CoV-2 Pneumonia in the Emergency Department. Ann Emerg Med. 2020 Oct 13:S0196-0644(20)31269-5. doi: 10.1016/j.annemergmed.2020.10.008. Epub ahead of print. PMID: 33461884; PMCID: PMC7552969.

13. Williamson EJ, Walker AJ, Bhaskaran $\mathrm{K}$ et al. Factors associated with COVID-19-related death using OpenSAFELY. Nature. 2020 Aug;584(7821):430-436. doi: 10.1038/s41586-020-2521-4. Epub $2020 \mathrm{Jul}$ 8. PMID: 32640463.

14. Siddiqi HK, Mehra MR. COVID-19 illness in native and immunosuppressed states: A clinicaltherapeutic staging proposal. J Heart Lung Transplant. 2020 May;39(5):405-407. doi: 10.1016/j.healun.2020.03.012. Epub 2020 Mar 20. PMID: 32362390; PMCID: PMC7118652.

15. Myrstad M, Ihle-Hansen H, Tveita AA et al. National Early Warning Score 2 (NEWS2) on admission predicts severe disease and in-hospital mortality from Covid-19 - a prospective cohort study. Scand J Trauma Resusc Emerg Med. 2020 Jul 13;28(1):66. doi: 10.1186/s13049-020-00764-3. PMID: 32660623; PMCID: PMC7356106.

16. Carr E, Bendayan R, Bean D et al. Evaluation and improvement of the National Early Warning Score (NEWS2) for COVID-19: a multi-hospital study. BMC Med. 2021 Jan 21;19(1):23. doi: 
10.1186/s12916-020-01893-3. PMID: 33472631; PMCID: PMC7817348.

17. Ruch $Y$, Kaeuffer $C$, Ohana $M$ et al. CT lung lesions as predictors of early death or ICU admission in COVID-19 patients. Clin Microbiol Infect. 2020 Oct;26(10):1417.e5-1417.e8. doi: 10.1016/j.cmi.2020.07.030. Epub 2020 Jul 24. PMID: 32717417; PMCID: PMC7378475.

18. Inui S, Fujikawa A, Jitsu M et al. Chest CT Findings in Cases from the Cruise Ship Diamond Princess with Coronavirus Disease (COVID-19) Radiology: Cardiothoracic Imaging 2020; 2(2):e200110 doi.org/10.1148/ryct.2020200110

19. Lichter Y, Topilsky Y, Taieb $P$ et al. Lung ultrasound predicts clinical course and outcomes in COVID19 patients. Intensive Care Med. 2020 Oct;46(10):1873-1883. doi: 10.1007/s00134-020-06212-1. Epub 2020 Aug 28.

20. COVID-19 Treatment Guidelines Panel. Coronavirus Disease 2019 (COVID-19) Treatment Guidelines. National Institutes of Health. Available at https://www.covid19treatmentguidelines.nih.gov/. Accessed February 2, 2021

\section{Figures}




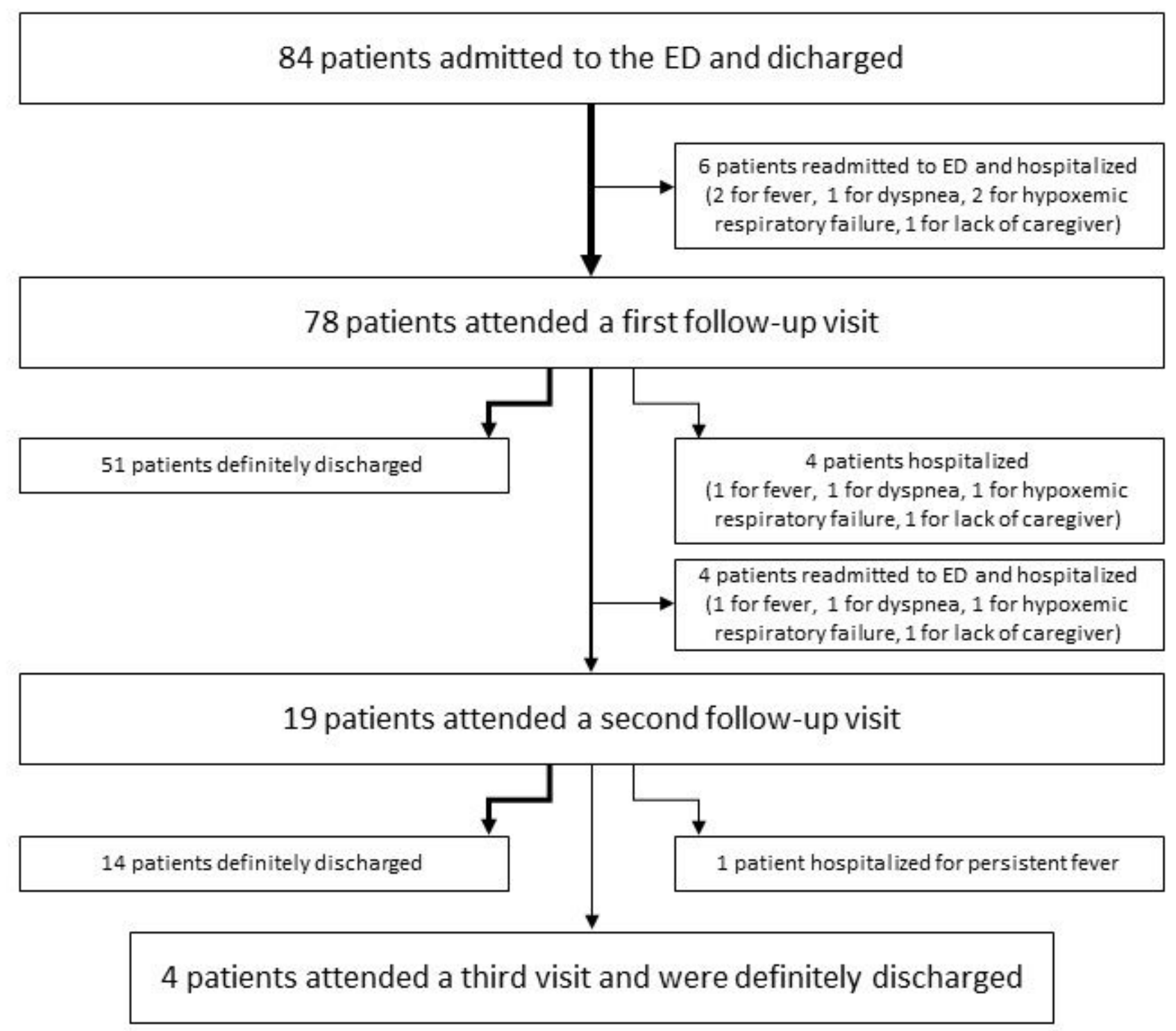

\section{Figure 1}

The flow-chart summarizes the management course of the enrolled patients: admission, revaluation or discharge. ED: Emergency Department. 


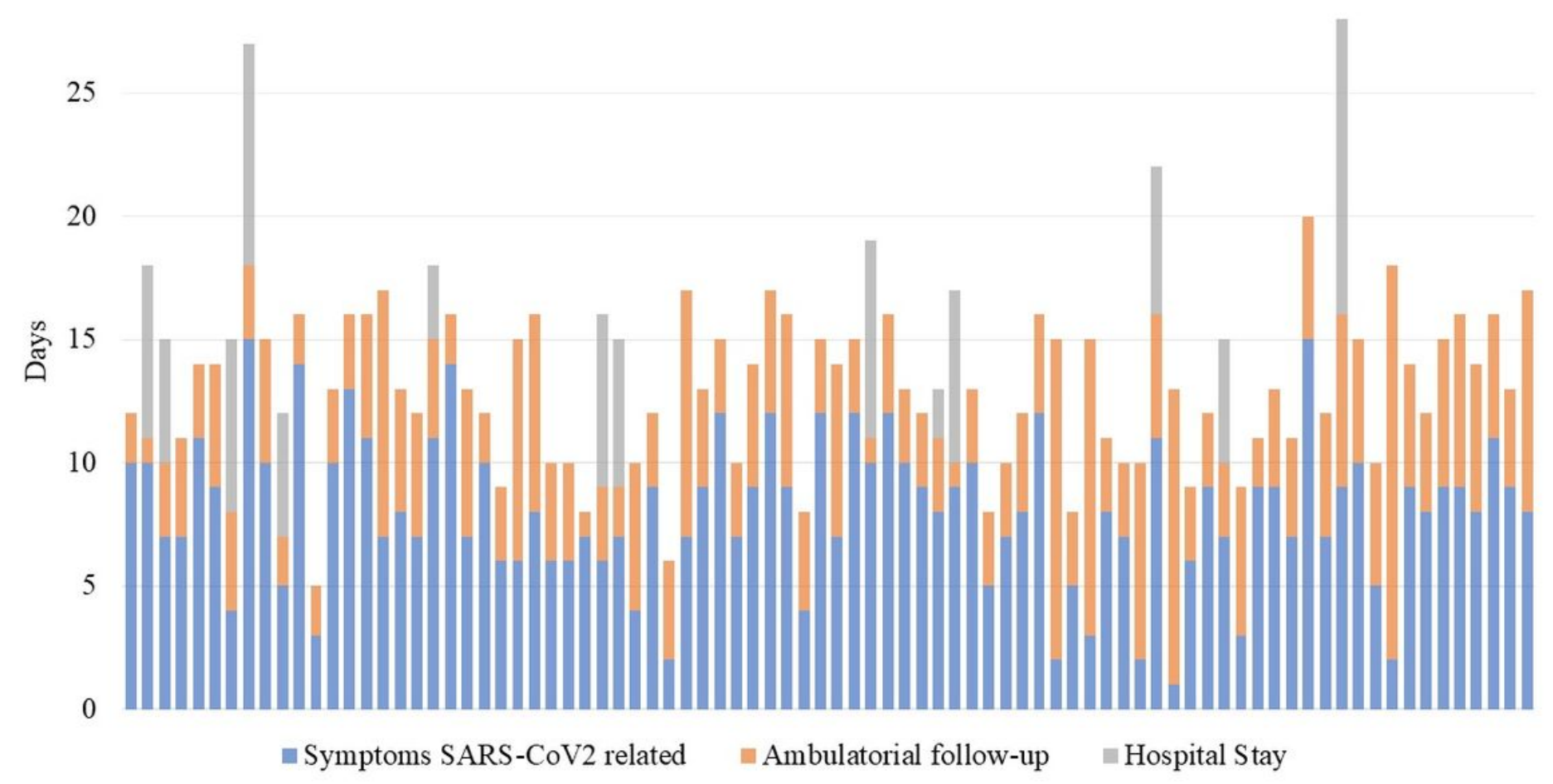

Figure 2

The graph shows the disease course of each patient from the onset of symptoms to the discharge or hospitalization. 